\title{
A Survey on Localization and Covering Techniques in Wireless Sensor Networks
}

\author{
Lokesh Poudyal \\ Sikkim Manipal Institute of Technology \\ Majitar, Rangpo \\ East Sikkim
}

\author{
Biswaraj Sen \\ Sikkim Manipal Institute of Technology \\ Majitar, Rangpo \\ East Sikkim
}

\begin{abstract}
Wireless Sensor Network (WSN) has gained popularity in diverse application fields owing to the inexpensive sensor nodes also called as motes. These sensor nodes are either deployed randomly or placed manually depending on the type of applications. The services of WSN are tremendously being used in application areas such as disaster relief, search and rescue, target tracking, battlefield and many more. Due to the application of WSN in such environments, it is not possible to manually deploy sensor nodes and therefore, the nodes are randomly deployed in the area of interest. Due to the random placement of the sensor nodes, it becomes essential to determine the location of the nodes in order to facilitate communication between nodes as well as Base Station (BS). Further, in case of random node deployment, the usual practice is to deploy a very large number of nodes (more than that of the requirement) in the area of interest. This is done in order to achieve fault tolerance, thus opening a large number of research directions to determine the optimal number of nodes participating in WSN 'covering' problem. This paper deals with an insight on the various localization and coverage techniques used in WSN. Further this paper tries to establish the interdependence between the localization and covering techniques.
\end{abstract}

\section{General Terms}

Sensor network, localization, coverage, base station.

\section{Keywords}

WSN, SERLOC, HIRLOC.

\section{INTRODUCTION}

Wireless Sensor Network (WSN) consists of a number of tiny nodes that are placed in an environment wherein they can sense the phenomena like temperature, sound etc [7].The sensor nodes passes the data from the source to the destination i.e. the Base Station (BS). The nodes in the WSN are generally deployed randomly. The sensor nodes have energy constraints in the form of limited source of power, limited memory, limited communication range $[7,4]$.The nodes will perform well until their power is utilized fully. They are termed as dead nodes when their energy is fully lost and unable to perform its task.WSN is very useful because its implementation is cheaper than that of a wired network and it is ideal for non-reachable places such as across the river, mountain or rural areas. On the other hand WSN has some drawbacks also like sensor nodes have resource constraint nature and are more difficult to configure than wired networks [2]. The WSN are used in the different application like traffic monitoring, battlefield, civilian, health care, monitoring of environment, wildlife, farming, fire detection etc [7]. Owing to the constraints imposed on the sensor nodes such as limited battery capacity, limited communication range, limited memory etc. several issues such as coverage, routing, security, aggregation, localization are to be addressed differently in WSN in comparison to wired networks. The main focus of this paper is the localization and coverage. The sensor nodes are deployed randomly in an environment to form the sensor network. Hundreds to several thousands of nodes are deployed throughout the sensor field to create a wireless multi-hop network. Nodes can be deployed as dense as 20 nodes $/ \mathrm{m} 3$ and as sparse as $3 \mathrm{~m}$ apart nodes [1,3,5 ]. After establishing the network to find the exact location $(\mathrm{x}, \mathrm{y}, \mathrm{z}$ coordinate of a particular location) of a sensor node in the environment is called localization, and it is one of the most challenging problems in WSN[6]. Further after deployment and localization, identification of the area covered by the particular sensor node is a challenging task and is termed as coverage. In order to identify the area coverage it is necessary to identify the location of the sensor node first, thus localization and coverage are inter-related to each other.

\subsection{WSN Communication Structure}

The major components of a typical sensor network are: sensor nodes, the sensor field, the sink and the Base Station (BS) as shown in Figure 1.

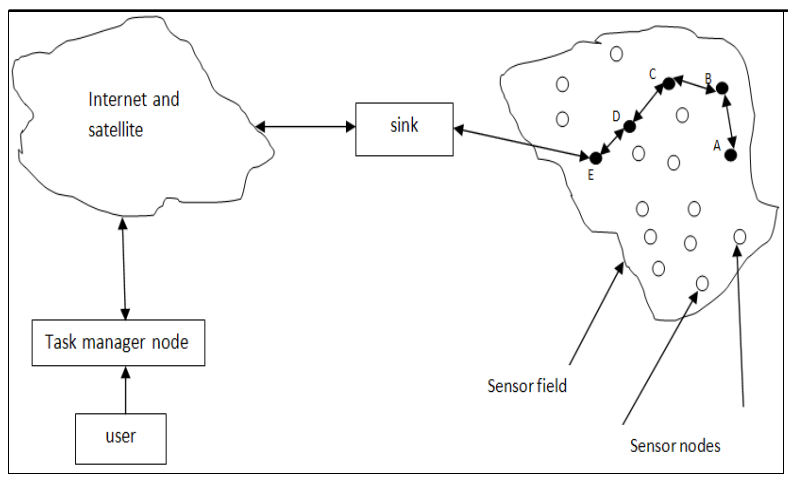

Fig 1: Components of a WSN [7]

The sensor nodes also called motes are the main components of WSN. They are very tiny devices having limited power resource, computation and communication range. They are assigned the crucial role of collecting data and routing this information back to a sink. Typically a sensor node consists of sensing, communication and processing module [10]. The sensing module is used to collect the data from the environment by sensing the environment, where as the communication module is used to communicate between two nodes. The processing module also called the data processing unit consists of small processor called microcontroller having low power consumption. Sensor nodes also have memory depending on the requirements for which the sensor node is 
used [7][10].Generally a battery is used to store power for sensing communication and data processing. A typical characteristic of sensor nodes is that the communication is more costly than computation. A sensor field can be considered as the area in which the nodes are placed i.e. the area in which we expect a particular phenomenon to occur. In order to reduce the total number of messages that need to be sent, some of the nodes are designated as the sink in order to reduce the overall energy requirements of the network. Hence a sink is one of the special sensor nodes with the specific task of receiving, processing and storing data from the other sensor nodes in the network. Any regular nodes can also be considered as sinks if they delay outgoing messages until they aggregate enough information. Thus sinks are called data aggregation points. The Base Station (BS) is a centralized point of control within the network and is a powerful data processing /storage centre. The main function of the BS is to collect information from the network and broadcast control information back into the network. Thus, it is also called the gateway to other networks[7].

\subsection{Issues in WSN}

WSN can be used in different applications like medical applications, battlefields, environmental and natural habitat monitoring, medical applications and detection of the forest fire etc [6]. Some of the issues of the WSN are Localization, Coverage, Deployment, Quality of Service (QoS), Routing, Security etc. The main objective of this survey paper is to study different protocols for the localization and coverage.

The remaining portion of the paper is organized as follows: In Section II some localization algorithm such as SERLOC, One hop localization and HIRLOC are discussed. Section III presents the Coverage analysis. Section IV, contains the conclusion.

\section{LOCALIZATION}

WSN consists of several sensor nodes. For all the applications of the WSN, the sensor nodes can be either hand deployed or randomly deployed $[1,5]$. In the latter case, finding the location of the nodes becomes necessary before initiating the communication process. The process of finding the exact location of the sensor nodes after its deployment in the environment is called localization $[1,4,10]$. The localization problem can be solved by the use of GPS. The main drawback of having GPS in all the sensor node is that the network will be very costly. Further, the role of GPS is vital only during the initial phases of the network setup process. Thus, its use results in increase in power consumption, thus reducing the battery life of the sensor nodes. This in turn reduces the effective lifetime of the entire network. Further the main architecture of the sensor node will be changed if equipped with GPS, thus increasing the production cost of the sensor node as a whole. In addition to this GPS signals are not available in some of the environmental conditions like underground water and so. All these factors results in a high cost network with extra overhead. The alternative to this is the usage of GPS in only few nodes called anchor or beacon nodes. These anchor nodes are special nodes having GPS attached on them, thus they know their location. Based on the known location of the anchor nodes, the location of all other nodes can be calculated [17]. The main advantage of this scheme is the usage of very few GPS based nodes, thus reducing the cost of the network setup. Various GPS free localization techniques have been proposed for measuring the distances in range-based algorithms [16] which are based on complicated mathematical formulations. Bischoff et al. [16] used received signal strength (RSS) to estimate distances between the nodes. The main drawback of this scheme is wastage of computational capacity, in solving the mathematical formulae, owing to the limited computational capacity of the sensor nodes. Since the knowledge of the node location can be used to implement energy efficient message routing protocols in sensor networks, the decision of using or not using GPS equipped nodes plays a vital role in minimizing the energy usage of the WSN. The precision of the localization increases with the number of beacons [17].However, the main problem with increased beacons is that they are more expensive than other sensor nodes because GPS consumes significant battery power thus can be a problem for power-constrained sensor nodes [17]. In addition to this, once the unknown stationary nodes have been localized using beacon nodes, the role of beacon nodes get over. Thus the selection of optimal number of beacon nodes also plays an important role in increasing the overall life of the network. Use of complicated mathematical formulations should be avoided as much as possible because the computation capacity of sensor nodes is also limited.

\subsection{Formulation of a Typical Localization Problem}

Some sensor nodes are aware of their own positions, these nodes are known as beacons. All the other nodes localize themselves with the help of location references received from the anchors. So, mathematically the localization problem can be formulated as follows: given a multihop network, represented by a graph $\mathrm{G}=(\mathrm{V}, \mathrm{E})$, and a set of beacon nodes $\mathrm{B}$, their positions $\{\mathrm{xb}, \mathrm{yb}\}$ for all $\mathrm{b} \varepsilon \mathrm{B}$, it is possible to find the position $\{\mathrm{xu}, \mathrm{yu}\}$ for all unknown nodes $\mathrm{u} \varepsilon \mathrm{U}$ where $\mathrm{xi}$ and $\mathrm{yi}$ are the $\mathrm{x}$ and $\mathrm{y}$ co-ordinates of a node $\mathrm{i}$.

\subsection{SEcure Range-independent Localization scheme (SERLOC)}

In WSN, the decentralized, resource-efficient sensor localization can be achieved by a novel range-independent localization scheme, called SERLOC [19].The focus of SERLOC is on secured localization. By using this localization scheme security can be achieved that allow each sensor to determine its location even in the presence of well known threats on WSN. SERLOC is robust against varying sources of error which localizes sensors with higher accuracy [19].

In SERLOC, the locators transmit the beacon information and based on that information, location can be determined by the sensors [7,19]. Each beacon information contains: the locator's coordinates and the angles of the antenna boundary lines, with respect to a common global axis. If a sensor hears a beacon transmitted at a specific antenna sector of a locator $\mathrm{Li}$, it has to be included within that sector. The sensors know the locators' communication range $\mathrm{R}$ and through the beacons, they acquire the locator's coordinates and sector boundary lines [11]. They can identify the region within which they reside by computing the overlap between all the sectors that they hear. Each sensor uses the center of gravity $(\mathrm{CoG})$ of the overlapping region which is used to determine its location through the beacon information. The sensor can lie with equal probability at any point of the overlapping region. The position of a sensor can be determined by using an algorithm which consists the following four steps.

Step 1: Collection of the beacons by the sensors from all locators it can hear.

Step 2: Determination of an approximate search area within which sensor can be located based on the coordinates of the locators heard. 
Step 3: Computation of the overlapping antenna sector region by using a majority vote scheme.

Step 4: Determination of sensor location as the CoG of the overlapping region.

\subsubsection{SERLOC Security Architecture}

The security mechanisms of SERLOC can be described by computing the secure location $[19,22]$. The stated mechanism will protect the localization information by encrypting all beacons which are transmitted from locators. There are different keying techniques which can be used but asymmetric key cryptography keying technique should not be used because there are some constraints associated with sensors, like computational power and energy resources [7,19,22]. Sensors and locators share a global symmetric key $\mathrm{K} 0$ and this key should be pre-loaded before deployment. In addition, every sensor shares a symmetric pairwise key KL with every locator Li. The shared key KL should also pre-loaded. Storage is also one of the issue in WSN therefore considering the storage constraints, the total size of above keys should be minimum and this can be achieved because the number of locators deployed is relatively small. The use of a shared symmetric key does not identify the source of the messages that each sensor hears and in the absence of additional security features, a malicious sensor may change the behavior of multiple locators. Therefore there should be some locator identification authentication technique. The false localization information into the network can be prevented by authenticating the source of the beacons using collisionresistant hash functions.

\subsection{One-Hop Localization Techniques[20,21]}

One-Hop Localization Techniques use measurement techniques in WSN which can be classified into three types [20]: Angle-of-Arrival (AOA) measurements distance based measurements and Received Signal Strength (RSS) profiling techniques. Distance based measurements are further classified into one-way propagation time and roundtrip propagation time measurements. The AOA measurement techniques can be further classified into two types: those making use of the response obtained by receiver antenna's amplitude and those making use of the phase response received by receiver antenna. Beam forming is the base of AOA measurement techniques which is used in the reception pattern of an antenna. In comparison with the wavelength of the signals, the unit for measurement will be small in size. Distance based measurements finds propagation time based measurements, i.e., one-way propagation time measurements, roundtrip propagation time measurements and Time Difference-Of-Arrival (TDOA) measurements, and RSS measurements [21]. There are some other distance measuring technique which does not fall into the above categories. The name of these techniques are One-way propagation time and round-trip propagation time measurements, Lighthouse approach to distance measurements , Time-difference-ofarrival measurements, Distance estimation via received signal strength measurements. The RSS profiling-based localization techniques to construct a map of the signal strength behavior in the coverage area. The map is obtained either by a priori measurement or using sniffing devices $[20,21]$ deployed at known locations. In this technique, in addition to there being anchor nodes which may be like an access point in wireless local area network and non-anchor nodes. Non-Anchor may be a large number of sample points which are distributed throughout the coverage area of the sensor network. Based on the jth entry corresponding to the jth anchor's transmitted signal, a vector of signal strengths can be obtained at each sample point. The map of the whole region can be obtained by collecting all these vectors. The map can also contain the RSS model which is unique with respect to the anchor locations and the environment. The model is stored in a central location. The RSS model is referred by a non-anchor node to estimate its location using the RSS measurements from anchors. Thus, there are many measurement techniques for WSN localization and which measurement technique should be used for location estimation, that depends on the specific applications of WSN $[5,20,21]$. The localization algorithms based on AOA and propagation time measurements are able to achieve better accuracy than localization algorithms based on RSS measurements.

\subsection{High - Resolution range-independent Localization scheme (HIRLOC) [18]}

The localization for WSN should be secured. For making the localization a secure, there should be: - a decentralized and a scalable implementation of the proposed technique for localization, resource efficiency, computation, communication and storage, range-independence, and robustness against security threats in WSN[18]. The High resolution Rangeindependent Localization (HIRLOC) allows sensors to passively determine their location with high accuracy. Passively in the sense that the sensors do not interact to determine their location. The multiple localization information can be combined together over a short time period so that the accuracy of the localization can be increased [23]. In this scheme, the increase in the expense in the hardware will be very less and, and the reference points are deployed with higher density. The HIRLOC technique is not susceptible to any range measurement alteration attacks because it does not perform any range measurements to estimate the sensor's location. Furthermore, sensors do not rely on other sensors to infer their location and hence, the robustness of this technique does not rely on the easily tampered sensor devices but it is robust against well known security threats in WSN, such as the wormhole attack, the Sybil attack and compromise of network entities. Based on the performance evaluation, it has been seen that HIRLOC localizes sensors with higher resolution than decentralized range-independent localization schemes and it requires less hardware resources. It is assumed that sensors are equipped with omnidirectional antennas, able to transmit with maximum power [7, 24].

\subsubsection{Location Determination}

The locators transmit the beacon information to the sensors to determine their location [18]. The beacon information contains, (a) the locator's coordinates, (b) the angles of the sector boundary lines defined by the directional transmission, with respect to a common global axis and, (c) the locator's communication range $\mathrm{R}$. The locators may retransmit beacons in order to improve the accuracy of the location estimate. The retransmission can be possible by changing the orientation of a locator over time. Based on the beacon information, sensors define the sector area $\mathrm{Si}(\mathrm{j})$ as the confined area covered by the jth transmission of a locator $\mathrm{Li}$. The sector area includes jth beacon transmission from locator Li. HIRLOC uses some methods for reducing the ROI by exploiting the temporal dimension and the costs of deploying more locators are very less. The locators provide different localization information at consecutive beacon transmissions by $[18,24]$ :- 
(a) Changing the direction of their antennas:- Using multiple directional antennas, locator transmits in all directions for the Omni directional coverage

(b) Changing the communication range of the transmission via power control:- This approach is used to reduce the area of the ROI, i.e. to reduce the size of the intersecting sectors.

(c) Hybrid approach: The combination of the methods described in (a) and (b) leads to a dual dependency of the sector area $S i(\mu i(j) ; R(j) ; j)$ : Such a dependency can also be interpreted as a limited mobility model for the locators.

The ROI can be reduced by using these methods. The methods uses:-

\subsubsection{The algorithmic details of HIRLOC}

The HIRLOC technique uses the following two steps [18]:-

Step1:- Computing the intersection of all sector areas

Step 1.1:- Initial estimate of the ROI

Step 1.2:- Beacon collection

Step 1.3:- Determination of the ROI

Step 2:- Computing the sector intersection at each transmission round

\section{COVERAGE}

In case of dense network the coverage of the particular sensor node can be identified based on node's localization information. In other words, in order to have identification of nodes, which is necessary for coverage, the exact position of all sensor nodes in the environment are required. Therefore for proper coverage, localization of the sensor nodes are also required. The different algorithms to solve the problem of localization are discussed in [10].

The sensor nodes are deployed in an environment to perform a particular task like sensing, communication and computation. At the time of deployment, it is required to find out the area covered by the typical sensor node. The main aim of the Coverage problem is to determine the area covered by the sensor node in a particular environment / deployment area. A large number of research papers have been reported covering the concepts and solutions to the WSN converge problems $[6,7,10]$. Zhang and Hou [12] proposed a solution to the problem of coverage for large-scale sensor networks. The authors investigated the relation between coverage and connectivity and proved that if the communication range is at least twice the sensing range then complete coverage of an area guarantees a connected network [12]. Tian and Georganas [13] proposed a self-scheduling scheme that can reduce overall energy consumption by allowing each node to decide whether to turn itself off or on using only local neighborhood information. Gao, et al. [14] proved that at least three and at most five one-hop neighbors are needed to cover the whole sensing area of node si.

As proposed by Gage[3] there are basically three types of coverage as. They are:

Blanket Coverage: Coverage of an entire area is known as full coverage, popularly known as blanket coverage. It is used to check whether every single point is within the field of interest i.e. within the sensing range of at least one sensor node or not.
Barrier coverage: It refers to the detection of movement across a barrier of sensors [10] or boundary area.

Sweep Coverage: It is similar to that of barrier coverage but it addresses the moving target as in case of enemy movement in a battlefield surveillance area.

Apart from location information of the nodes, in order to determine the coverage area it is necessary to find the Coverage Percentage (CP). Coverage percentage is defined as the ratio of the area covered by the sensor node to the total number of the sensor nodes that are deployed in the area of interest [8].

$(\mathrm{CP})=$ No of nodes covered by a particular node / total no of nodes $\times 10000$

Due to the limited power available to the sensor node, these nodes need not be active all the times. Based on this fact, it is necessary to minimize the number of nodes participating in the communication. This leads to an open research area of finding the optimal number of active sensor nodes.

\section{CONCLUSION}

In this paper, different localization techniques of WSN are discussed. By using SERLOC technique, decentralized, resource-efficient sensor localization can be achieved and security can be provided. One-Hop Localization Techniques use measurement techniques and on the other hand HiRLoc localization technique allows sensors to passively determine their location with high accuracy and this technique is resource efficient in computation, communication and storage, range-independence, and robustness against security threats in WSN. This technique also reduces the ROI. The different coverage technique like blanket coverage, sweep coverage and barrier coverage, along with the method to calculate the coverage percentage are also discussed in this paper.

\section{ACKNOWLEDGMENTS}

The authors would like to thank the Head of the department and the faculty members of Computer Science and Engineering Department of Sikkim Manipal Institute of Technology for their useful suggestion in the right direction.

\section{REFERENCES}

[1] Pal. 2010. "Localization Algorithms in Wireless Sensor Networks: Current Approaches and Future Challenges", Network Protocols and Algorithms, ISSN 1943-3581, Vol. 2, No. 1.

[2] Ghosh and S. K. DAS. 2006. 'Coverage and Connectivity Issues in Wireless Sensor Networks',Mobile, Wireless, and Sensor Networks: Technology, Applications, and Future Directions, Akkihebbal L. Ananda, Mun Choon Chan, and Wei Tsang Doi Copyright, John Wiley \& Sons, Inc, pp:221-256,

[3] D. W. Gage. 1992. Command control for many-robot systems, Proc. 19th Annual AUVS Technical Symp. Reprinted in Unmanned Syst. Mag. 10(4):28-34.

[4] D. G. Anand, H. G. Chandrakanthand M. N. Giriprasad. 2011. "Energy Efficient Coverage Problems in Wireless Ad Hoc Sensor Networks", Advanced Computing: An International Journal(ACIJ), Vol No .2, , DOI : 10.5121/acij.2011.2204, pp:42-50. 
[5] E. Shih, S. Cho, N. Ickes, R. Min, A. Sinha, A. Wang, A.Chandrakasan. 2001. "Physical layer driven protocol and algorithm design for energy-efficient wireless sensor networks",In Proceedings of ACM MobiCom'01, Rome, Italy, pp. 272-286.

[6] G. J. Fan and ShiYao Jin. 2010. "Coverage Problem in Wireless Sensor Network:A Survey", Journal of Networks, VOL. 5, NO. 9 ,pp:1033-1041.

[7] G. Shankar , T.G.Basavaraju , D.H.Manjaiah, S. K.Sarkar. (July $2-4,2008$ ). "Issues in Wireless Sensor Networks", In Proceedings of the World Congress on Engineering Vol I WCE (July 2 - 4, 2008), London, U.K., ISBN:978-988-98671-9-5.

[8] I.F. Akyildiz, W. Su, Y. Sankarasubramaniam, E. Cayirci. 2002. "Wireless sensor networks: a survey,Computer Networks", Published by Elsevier Science B.V. PII: S13 8 9-1 286 (0 1 )0 030 2- 4, pp: $393-422$.

[9] J. Norman. 2011. "Connectivity and Coverage in Hybrid Wireless Sensor Networks using Dynamic Random Geometric Graph Model", International journal on applications of graph theory in wireless ad hoc networks and sensor networks (GRAPH-HOC) Vol.3, No.3, DOI : 10.5121/jgraphoc.3304 39,pp:39-47.

[10] R. Mulligan and H. M. Ammari. 2010. "Coverage in Wireless Sensor Networks: A Survey,Network Protocols and Algorithms", ISSN 1943-3581, Vol. 2, No. 2,pp: 2753.

[11] S.Gandhama, M.Dawandeb, R.Prakash. 2008. "Link scheduling in wireless sensor networks: Distributed edge-coloring revisited", J. Parallel Distrib. Comput. 68, doi:10.1016/j.jpdc.2007.12.006, pp:1122-1134.

[12] H. Zhang and J. C. Hou. 2004. Maintaining sensing coverage and connectivity in large sensor networks, Proc. Int. Workshop on Theoretical and Algorithmic Aspects of Sensor, Ad Hoc Wireless and Peer-to-Peer Networks (AlgoSensors), Florida.

[13] D. Tian and N. D. Georganas. 2002. A coveragepreserving node scheduling scheme for large wireless sensor networks, Proc. 1st ACMInt. Workshop onWireless Sensor Networks and Applications (WSNA'02), Atlanta, GA, pp. 32-41.
[14] Y. Gao, K. Wu, and F. Li. 2003. Analysis on the redundancy of wireless sensor networks, Proc. 2nd ACM Int. Conf. Wireless Sensor Networks and Applications (WSNA’03), San Diego, CA, pp. 108-114.

[15] G.M. Crippen, T.F. Havel. 1988. Distance Geometry and Molecular Conformation, John Wiley and Sons Inc., New York.

[16] U. Bischoff, M. Strohbach, M. Hazas, and G. Kortuem. 2006. Constraint-based distance estimation in ad-hoc wireless sensor networks. In Proceedings of the Third European Workshop on Wireless Sensor Networks (EWSN), pp. 54-68.

[17] J. Bachrach and C. Taylor,Localization in sensor networks. 2005. " in Handbook of Sensor Networks (I. Stojmenovic,ed.), pp: 1-38.

[18] Loukas Lazos and Radha Poovendran. 2005. "HiRLoc: High-resolution Robust Localization for Wireless Sensor Networks", OMB No. 0704-0188, pp 1-15.

[19] Loukas Lazos and Radha Poovendran. 2005. "SeRLoc: Secure Range-Independent Localization for Wireless Sensor Networks", WiSe'04, Philadelphia, Pennsylvania, ACM Transactions on Sensor Networks, Vol. 1, No. 1, pp. $73-100$

[20] D. J. Torrieri, "Statistical theory of passive location systems. 1984. " IEEE Transactions on Aerospace and Electronic Systems, vol. AES-20, no. 2, pp. 183-198.

[21] M. Gavish and A. J. Weiss. 1992. "Performance analysis of bearing-only target location algorithms," IEEE Transactions on Aerospace and Electronic Systems, vol. 28, no. 3, pp. 817-828.

[22] Y. Hu, A. Perrig, and D. Johnson. 2003. Packet Leashes : A Defense Against Wormhole Attacks in Wireless Ad Hoc Networks, In Proc. of INFOCOM, San Francisco, CA, USA.

[23] S. C $C^{\sim}$ apkun, J. Hubaux. 2005. Secure Positioning of Wireless Devices with Application to Sensor Networks, In Proceedings of the IEEE INFOCOM.

[24] P. Bahl and V. Padmanabhan. 2000. RADAR: An InBuilding RF-Based User Location and Tracking System, In Proceedings of the IEEE INFOCOM, Tel-Aviv, Israel, pp. 775-784. 